\title{
Evaluation of Geometric Features As Biomarkers of Diabetic Retinopathy for Characterizing the Retinal Vascular Changes During the Progression of Diabetes*
}

\author{
Georgios Leontidis, Member, IEEE, Bashir Al-Diri, Member, IEEE, Jeffrey Wigdahl, Member, IEEE, \\ and Andrew Hunter, Member, IEEE
}

\begin{abstract}
Diabetic retinopathy (DR) has been widely studied and characterized. However, until now, it is unclear how different features, extracted from the retinal vasculature, can be associated with the progression of diabetes and therefore become biomarkers of DR. In this study, a comprehensive analysis is presented, in which four groups were created, using eighty fundus images from twenty patients, who have progressed to DR and they had no history of any other diseases (e.g. hypertension or glaucoma). The significance of the following features was evaluated: widths, angles, branching coefficient (BC), angle-to-BC ratio, standard deviations, means and medians of widths and angles, fractal dimension (FD), lacunarity and FD-to-lacunarity ratio, using a mixed model analysis of variance (ANOVA) design. All the features were measured from the same junctions of each patient, using an automated tool. The discriminative power of these features was evaluated, using decision trees and random forests classifiers. Cross validation and out-of-bag error were used to evaluate the classifiers' performance, calculating the area under the ROC curve (AUC) and the classification error. Widths, FD and FDto-Lacunarity ratio were found to differ significantly. Random forests had a superior performance of 0.768 and 0.737 in the AUC for the two cases of classification, namely three-years-pre$D R /$ post-DR and two-years-pre-DR/post-DR respectively.
\end{abstract}

\section{INTRODUCTION}

Diabetic retinopathy (DR) is a major disease affecting millions of people around the world, leading even to blindness if not diagnosed early and taken care properly. Each human's retina is unique, like the fingerprint, thus it is important to understand how the disease affects each individual's eye during the progression of diabetes. The complications of diabetes can affect the retinal vasculature severely, thus it is required from diabetic patients to monitor regularly their retina for the appearance of any lesions [1].

The functional impairment during diabetes causes alterations in the blood flow and in the vessel wall structure, which can in turn affect the function of the retina, such as the endothelium and the blood retinal barrier [2-3]. These changes are believed to start affecting the vascular geometry early, before the first stages of DR. Until now no

*Research supported by a Marie Skłodowska-Curie grant from the European Commission in the framework of the REVAMMAD ITN (Initial Training Research network), Project number 316990.

G. Leontidis, B. Al-Diri and A. Hunter are with the Laboratory of Vision Engineering in the School of Computer Science at the University of Lincoln, LN67TS, Lincoln, UK, (e-mail: gleontidis@lincoln.ac.uk).

J. Wigdahl is with the Department of Information Engineering at the University of Padova, Via Gradenigo 6/B, Padova, Italy (e-mail: wigdahl@dei.unipd.it). comprehensive effort has been made, in order to study the effects that diabetes has to the geometry of the retina in patients that progressed to diabetic retinopathy, in multiple time intervals of diabetes. Moreover, the investigated features were limited, for specific periods of time, or using different population as control group that does not provide us with indications on how the retinal vasculature changes within each patient during diabetes [4-6].

In this study, data from twenty patients were used, analyzing in total eighty high-resolution fundus images $(2352 \times 1568$ pixels $)$ coming from a database of diabetic retinopathy screening in UK. Four groups were created, depending on the period that the images were taken $(3$ years before DR, 2 years before DR, 1 year before DR and 1st year of DR). The diversity of retina, which includes many small and large vessels, creates many problems when it comes to compare the effects of diabetes in different cohorts. Choosing at random segments to measure, from different groups, and not being consistent on selecting a representative amount of large and small vessels, affects the final statistical comparison, since the samples have not been collected consistently, failing to represent reliably the unobserved population, increasing the statistical error.

In the proposed approach, the same segments are matched in all of the four groups, ensuring that the measurements include the same errors throughout, enhancing the statistical power of the study and therefore concluding with more meaningful results. To our best of knowledge this is the first time that such a comprehensive study is attempted.

\section{METHODS}

\section{A. Background}

In literature, various methods have been proposed for segmenting and measuring the retinal vasculature. Wang et al. presented a supervised method for segmenting retinal images, using ensemble learning, by combining a convolutional neural networks and random forests classifier [7]. Roychowdhury et al. implemented an unsupervised segmentation algorithm, identifying the vessels iteratively, by using an adaptive threshold and a region growing approach with a novel stopping criterion for the iteration [8]. Zhao et al. developed an infinite active contour model, using an infinite perimeter regularizer and multiple region information [9]. Lowell et al. presented a two dimensional Gaussian model, which fits a two-dimensional intensity vessel segment presenting a sub pixel accuracy [10]. Bankhead et al. used wavelets and edge location refinement both to segment and measure retinal vessels using image profiles, computed across a spline fit of each detected centerline [11]. 


\section{B. Tools}

From each of the eighty images, eight junctions (four veins and four arteries; in total 1920 widths and 640 angles) with the best quality were chosen, making sure that they can be identified and measured equally in a reliable manner in all of the progressed images, for every group and every patient. For each junction, the widths (in pixels) of the parent and children vessels and the angle (in degrees) were measured using a fully automated algorithm, based on an active contour model, which has been tested for its accuracy, reliability and robustness [12]. This algorithm, which was also used for segmenting the retinal vasculature, uses two pairs of contours to find the vessel edge, followed by a generalized morphological order filter that identifies the vessel centerline, and a neural cost function to resolve junction configurations.

In addition, the branching coefficient was calculated for every junction, and also the ratio between the angle and the branching coefficient (BC) was evaluated, in order to have a full description of every junction. This feature could possibly identify changes in the vessels' junctions as a whole, giving us an indication of the adaptation of the junctions during the progression of diabetes. Furthermore, the vascular trees were segmented, adjusting the algorithm accordingly in order to have the same amount of vessels in all of the images of the same patient, and thus calculate the fractal dimension accurately. An example of the segmentation from a patient the year before DR and the first year of DR, can be seen in Fig.1.

Fractals are complex patterns that exhibit self-similarity across different scales, giving us a measure of complexity. Human retina has been found to approximately have a selfsimilar structure in different scales, which means that it can be analyzed as a fractal structure [13]. This could give us an indication of whether the complexity of the vasculature changes with the progression of diabetes. Higher FD defines a more complex structure.

Fractality and lacunarity are complementary of each other and could give an index of the complexity of the vasculature and the gaps in the structure of the vessels. Lacunarity is a counterpart of fractality that actually describes how patterns like fractals fill the space in a way that if the fractal has large gaps, it presents high lacunarity; on the other hand if it is almost translationally invariant then it has low lacunarity, so it can be thought as a measure of gappiness or heterogeneity.

The fractal dimension was calculated using the boxcounting algorithm (Minkowski-Bouligand dimension) based on (1),

$$
D_{b}=\lim _{r \rightarrow 0} \frac{\log N(r)}{\log 1 / r}
$$
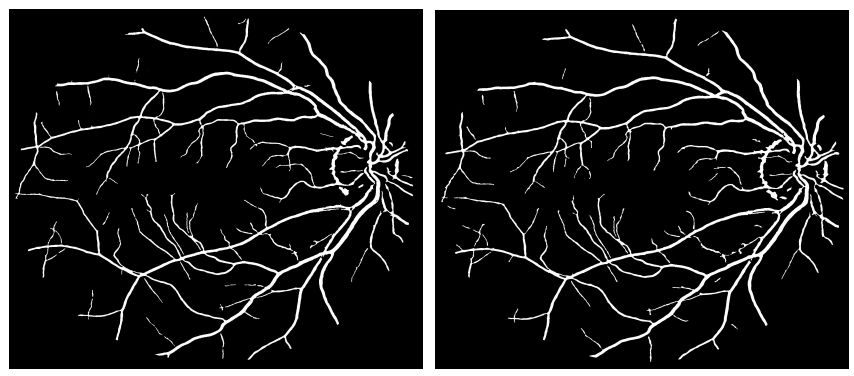

Figure 1. Two segmented images from the same patients for the calculation of FD and lacunarity. On the left we can see the year before DR and on the right the first year of DR. where $N_{r}$ is the number of boxes of side length $r$ that is needed to cover a given image in the Euclidean $n$-space, using a sequential number of descending sizes boxes in multiple orientations. The final dimension is between 1 and 2 $(1 \leq \mathrm{D} \leq 2)[14]$.

Lacunarity is calculated for different grid orientations using the gliding box algorithm, in a similar way like the box counting dimension. In this case we choose randomly a unit box of size $r$ and we count the number of set points $p$ within this box, namely the mass. Following that, the procedure is repeated with the box centered consecutively for each point within the set, creating a distribution of box masses $B(p, r)$ that refers to the number of boxes with $p$ points and radius $r$. This distribution is then converted into a probability distribution $Q(p, r)$, by diving by the total number of boxes (B) of size $r(2)$.

$$
\mathrm{Q}_{\mathrm{p}, \mathrm{r}}=\frac{\mathrm{B}_{(\mathrm{p}, \mathrm{r})}}{\mathrm{B}_{(\mathrm{r})}}
$$

After several transformations the gliding box lacunarity equation (3) can be written in terms of the accumulated sum of the mean and the second moment over all boxes,

$$
\operatorname{LGB}_{G}(\mathrm{r})=\frac{\mathrm{B}_{(\mathrm{r})} \sum_{\mathrm{i}=1}^{\mathrm{B}(\mathrm{r})} \mathrm{p}(\mathrm{i}, \mathrm{r})^{2}}{\left[\sum_{\mathrm{i}=1}^{\mathrm{B}(\mathrm{r})} \mathrm{p}(\mathrm{i}, \mathrm{r})\right]^{2}}
$$

where the denominator is the square of the total number of elements in the data set [15].

\section{Features}

The features that were investigated are the followings: parent and children widths separately and together, angles, measures of central tendency (mean and median values), measures of dispersion (standard deviations), fractal dimension, lacunarity and the ratio of the last two. In addition the branching coefficient was calculated, based on (4), as well as the ratio of the angle with the corresponding $\mathrm{BC}$, in order to give us an index of the junction.

$$
\mathrm{BC}=\frac{\mathrm{W}_{1}^{2}+\mathrm{w}_{2}{ }^{2}}{\mathrm{w}_{0}^{2}}
$$

Where w1, w2, w0 are the widths of the larger child vessel, smaller child vessel and parent vessel respectively. All of the features were evaluated using a mixed model analysis of variance (ANOVA) design implemented in Mathworks Matlab 2014b software, which takes into account the way that the measurements are taken (matched junctions) and also that the patients are the same among the four groups.

\section{DESIGN}

\section{A. Data structure}

The mixed model, which is based on the repeated measures nature of the analysis, usually increases the statistical power, requiring fewer subjects to be analyzed [16]. Including matched junctions and the same groups of patients, could lead to the decrease of both the statistical error (difference from the unobserved population mean) and the residuals (difference from the sample mean), which are usually higher when the groups are different. Using the same 
groups, we minimize the problems that cannot be easily standardized and might affect the analysis e.g. age, other diseases, gender, smoking etc., which have been shown to affect the retinal vasculature [17]. The proposed approach tries to minimize the two types of errors, by including the same conditions between and within the four groups, and also include all of the measurements as seen in Fig. 2, which shows the model having two groups just for illustration purposes. The cases, which refer to the four different groups, and the measurements, are the fixed factors, whereas the patients are the random factor since they are a sample of a larger population. The validity of the proposed model was tested to see if we get a uniform distribution on the histogram of the p-values, which we did, instead of the pvalues being pushed sideways.

\section{B. Statistics}

All of the features were analyzed using the design of Fig. 2. Each of the features represents the dependent variable, whereas the four different cases represent the independent variable. In order to use the ANOVA parametric test, instead of a non-parametric one, we have to make sure that the dependent variable is approximately normally distributed, even though it is robust in slight violations of normality. Another important assumption for the repeated measures is the sphericity, which refers to the fact that the variance of the population difference scores for any two conditions should be the same as the variance of the population difference scores for any other two conditions. A possible violation causes the test to become unstable (i.e., leads to an increase in the Type I error; that is, the likelihood of detecting a statistically significant result when there is not one) [18].

The Anderson-Darling and Shapiro-Wilk tests were used for testing for normality of each feature. The null hypothesis that the data are normally distributed was not rejected in either of the tests regardless of the feature under investigation. The p-values ranged from $0.33-0.57$ for the Anderson-Darling test and 0.40-0.61 for the Shapiro-Wilk test. Regarding sphericity, Mauchly's test was used, which again failed to reject the null hypothesis that the assumption of sphericity is met (p-values ranged from 0.11-0.35). In addition, for the significant results, post-hoc comparisons between the different groups were made, so as to identify between which two groups we have significant differences.

\section{Classifier}

Despite the limited amount of data, a random forests classifier was trained: a) for the pair three-years-preDR/first-year-of-DR and b) for the pair two-years-preDR/first-year-of-DR. Random forests classifier uses tree predictors in a way that each tree depends on the values of a

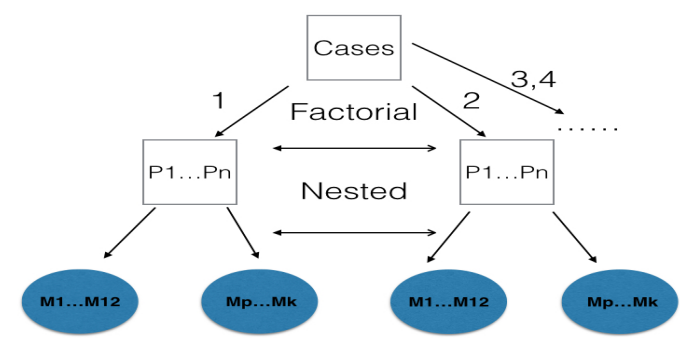

Figure 2. Mixed model used for the statistical analysis of each feature. random vector sampled independently keeping the same distribution across all the trees in the forest [19]. For this purpose, the freely available $\mathrm{R}$ software was employed for training the classifier, using 1000 trees and 10 features in total. It is worth mentioning that using all of the features did not affect significantly the performance of the classifier, apart from increasing slightly the out-of-bag error.

Regarding the parameter of the number of variables randomly sampled as candidates at each split, it was left to the default, since it appeared to have the best performance over selecting different values between two and ten, without affecting the error or the running time [20]. For evaluating the performance of the classifier the leave-one out cross validation was chosen, calculating the area under the ROC curve (AUC) and the result was compared with the out-ofbag error $(\mathrm{OOB})$, which is the internal method of validating the performance of a random forests classifier [19].

\section{RESUlTS AND DiscUSSION}

\section{A. Evaluation of Features}

In table 1 , the results of the analysis can be found, using all of the four groups. As can be seen, some of the features differed significantly across the groups, whereas some others not. In addition to that, no significant results (thus excluded from table 1) were observed in any combination of features when using the mean values, medians or standard deviations (although p-values were between 0.10-0.25), highlighting the superiority of using the proposed mixed model, in which all the measurements are accounted for as measured.

\section{TABLE I. SUMMARY OF THE FEATURES IN THE 4 GROUPS}

\begin{tabular}{|c|c|c|c|}
\hline \multirow[b]{2}{*}{ Features Names } & \multicolumn{3}{|c|}{ Mixed Model Analysis of Variance Results } \\
\hline & $\begin{array}{l}\text {-values } \\
(a=0.05)\end{array}$ & $\begin{array}{l}\text { F-values } \\
\text { (dfn, dfe) }\end{array}$ & $\begin{array}{c}\text { Group means }(S D) \\
(3 y, 2 y, 1 y, D R)\end{array}$ \\
\hline Artery' widths all & 0.0028 & $4.75(3,717)$ & $\begin{array}{l}9.17(0.70), 9.49(0.62), \\
9.35(0.63), 8.97(0.5)\end{array}$ \\
\hline Artery' widths par. & 0.038 & $2.86(3,237)$ & $\begin{array}{l}10.91(1.01), 11.19(0.82), \\
10.95(0.92), 10.43(0.75)\end{array}$ \\
\hline Artery' widths ch1 & 0.38 & $1.01(3,237)$ & $\begin{array}{l}8.14(0.95), 8.39(0.97), \\
8.45(1.05), 8.06(0.96)\end{array}$ \\
\hline Artery' widths ch2 & 0.12 & $1.93(3,237)$ & $\begin{array}{l}8.45(0.98), 8.89(0.86), \\
8.67(0.97), 8.41(0.99)\end{array}$ \\
\hline Artery' BC & 0.58 & $0.65(3,237)$ & $\begin{array}{l}1.24(0.13), 1.27(0.11) \\
1.29(0.14), 1.30(0.11)\end{array}$ \\
\hline Artery' angles & 0.79 & $0.34(3,237)$ & $\begin{array}{l}84.42(4.82), 83.53(3.79), \\
84.2(4.83), 84.58(4.30)\end{array}$ \\
\hline Artery' angle/BC & 0.55 & $0.7(3,237)$ & $\begin{array}{l}72.03(5.30), 69.11(6.51), \\
69.50(5.76), 68.25(8.78)\end{array}$ \\
\hline Veins' widths all & 0.0008 & $5.71(3,717)$ & $\begin{array}{l}11.58(0.67), 11.66(0.75), \\
11.28(0.62), 11.16(0.54)\end{array}$ \\
\hline Veins' widths par. & 0.04 & $3.1(3,237)$ & $\begin{array}{l}14.17(1.02), 14.05(1.40), \\
13.79(1.05), 13.6(1.17)\end{array}$ \\
\hline Veins' widths ch.1 & 0.05 & $2.9(3,237)$ & $\begin{array}{l}10.57(0.86), 10.55(1.17), \\
10.05(1.32), 10.1(0.92)\end{array}$ \\
\hline Veins' widths ch.2 & 0.2 & $1.5(3,237)$ & $\begin{array}{l}10(0.88), 10.25(1.01), \\
9.75(0.92), 9.87(0.95)\end{array}$ \\
\hline Veins' BC & 0.583 & $0.65(3,237)$ & $\begin{array}{l}1.12(0.10), 1.14(0.14), \\
1.11(0.15), 1.12(0.08)\end{array}$ \\
\hline Veins' angles & 0.85 & $0.26(3,237)$ & $\begin{array}{l}82.45(3.65), 81.70(3.1), \\
81.80(3.32), 82.01(3.45)\end{array}$ \\
\hline Veins' angle/BC & 0.51 & $0.77(3,237)$ & $\begin{array}{l}77.58(9.18), 74.19(11.1) \\
77.58(9.67), 75.3(7.8)\end{array}$ \\
\hline Fractal dimension & 0.04 & $3.02(3,57)$ & $\begin{array}{l}1.6253(0.07), 1.6191(0.06), \\
1.6076(0.05), 1.595(0.06)\end{array}$ \\
\hline Lacunarity & 0.77 & $0.37(3,57)$ & $\begin{array}{l}0.21(0.04), 0.20(0.04), \\
0.205(0.03), 0.22(0.05)\end{array}$ \\
\hline FD/Lacunarity & 0.041 & $2.99(3,57)$ & $\begin{array}{l}10.25(3.94), 9.86(4.1), \\
8.51(2.9), 8.57(3.40)\end{array}$ \\
\hline
\end{tabular}


In addition to the features in table 1 , which is a summary of the comparison of all the groups, the pairwise post-hoc comparison gave significant results when we compared the groups three-years-pre-DR/first-year-of-DR (p-values ranged from 0.001 to 0.04 ) and two-years-pre-DR/first-yearof-DR (p-values ranged from 0.006 to 0.05 ) for all the combinations of features that appear as significant in table 1. On the contrary, for the three-years-pre-DR/first-year-preDR the only significant result was observed for the veins' widths (parent $\&$ children together).

No significant results were observed for any features, when we compared either the pair first-year-pre-DR/firstyear-of-DR, which comes almost in line with the early results presented in previous study [6], or the pair of threeyears-pre-DR/two-years-pre-DR.

The values of the $\mathrm{BC}$ were close to the reported values in literature, although in our case the values refer to diabetic/DR patients whereas in literature for normotensive subjects [21]. These values could be used as an alternative way of calculating the arteriovenous ratio from the central retinal vein and artery equivalents, when the subjects do not belong to a no-disease group.

The asymmetry index $\gamma(5)$, defined as the ratio between the two children vessels, was calculated as well, which describes the relative relationship between the children vessels [22]. However neither statistical significance was found nor it contributed to the performance of the classifier.

$$
\gamma=\frac{\mathrm{w}_{1}^{2}}{\mathrm{w}_{2}^{2}}
$$

Where $\mathrm{w}_{1}$ and $\mathrm{w}_{2}$ are the two children vessels' widths.

Furthermore, the Pearson's correlation between the BC and angles, and also between the fractality and lacunarity were measured. No correlation was observed between the change of $\mathrm{BC}$ and the angles, whereas there was a negative significant correlation ranging from -0.93 to -0.96 between the fractality and lacunarity in each of the four groups (an example can be seen in Fig.3).

\section{B. Classification}

The classifier was built with a balanced design, without missing values, including 10 features with 40 observations per feature (20 for each class). The image-level (global)

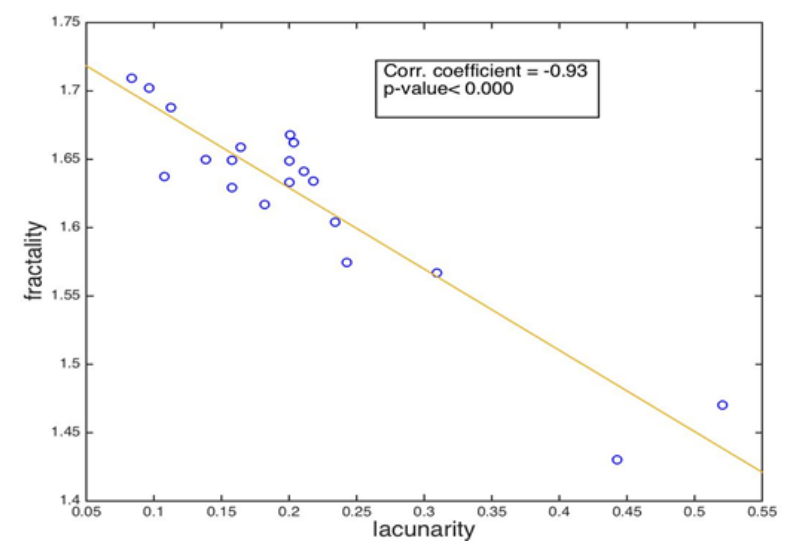

Figure 3. Plot of the correlation between fractal dimension and lacunarity at the third year before DR. features of FD, FD-to-lacunarity ratio, mean and standard deviation values of widths, $\mathrm{BC}$ and angle-to-BC ratios for both arteries and veins were used. The classifier had inferior performance when all the original values for arteries and veins were used instead of the descriptive statistics; therefore the aforementioned balanced structure was chosen.

As far as the group three-years-pre-DR/first-year-of-DR is concerned the AUC was 0.768 (values of sensitivity $=75.5 \% \&$ specificity $=63.4 \%$ ), whereas the group two-years-pre-DR/first-year-of-DR was 0.737 (values of sensitivity $=72.3 \% \&$ specificity $=61.1 \%$ ) (Fig.4). The OOB error was $24.6 \%$ and $26.1 \%$ respectively. Running a similar implementation in Matlab by training decision trees classifier, concluded with approximately the same results, presenting similar classification error. In the decision trees the resubstitution error is lower than the classification error because it is calculated using the same dataset for training and test, so it is quite biased, thus we mainly rely on the cross-validation error to evaluate the classifier. The running time to train the classifier in $\mathrm{R}$ was $0.24 \mathrm{~s}$, using a simple personal computer.

It is worth mentioning that the bootstrap aggregating technique that random forests classifier is using, leads to a better model performance, due to the decrease of the variance of the model, without seriously affecting the bias. In random forests, the subset of the features that are selected for building the ensemble model of decision trees, at each candidate split during the learning process is random.

\section{CONCLUSION}

To our best of knowledge, it is the first time that such a study is attempted, using four groups of the same progressed patients, both in terms of the analysis and the binary classification. Moreover, despite the small sample, the classifier had a fair performance in both cases, which means that probably with a larger sample its performance could improve. Some of the investigated features, like the widths, FD and FD-to-lacunarity ratio, could be used in the future as biomarkers of progression of diabetes, subject to further investigation. In this case the biomarkers could have a direct application by assigning higher risks to some patients and possibly help clinicians to examine these cases further. It is worth pointing out that decreased fractal dimension implies a less complex vasculature and also indicates alterations in the vessels. Another important observation is that in the significant features the mean values are smaller in the DR group, which can have various explanations, including the
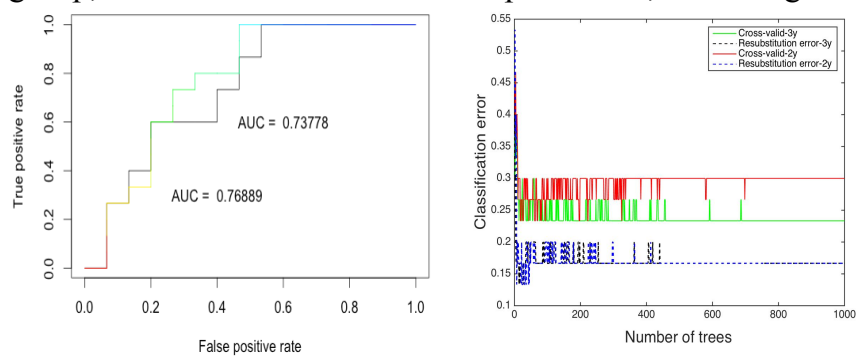

Figure 4. On the left we see the ROC plot showing the AUC values from random forests (cut-off $=0.5$ ). On the right, we can see the plots for the classification errors from the similar implementation with decision trees. 
constriction of the vessels to decrease the blood flow according to the local metabolic needs of the tissues.

The changes in the retinal vasculature during the progression of diabetes are severe, but due to the various sizes of vessels and the dynamic nature of the retina, where the vessels naturally adapt, makes it difficult to associate an alteration with diabetes or DR. In this direction, our immediate future work includes the investigation of additional features that can reliably be associated with the progression of diabetes, like lengths of matched segments, arteriovenous ratio, branching patterns, etc.

\section{ACKNOWLEDGMENT}

Many thanks to Evi kotsiliti from Health intelligence company in UK for providing the images for analysis and Phil Assheton for his valuable advice on the data analysis.

\section{REFERENCES}

[1] D. A. Antonetti, A. J. Barber, S. K. Bronson, W. M. Freeman, T. W. Gardner, L. S. Jefferson and I. A. Simpson, "Diabetic retinopathy seeing beyond glucose-induced microvascular disease," in Diabetes, vol.55, pp. 2401-2411, 2006.

[2] J. E. Grunwald, J. DuPont and E. R. Riva, "Retinal haemodynamics in patients with early diabetes mellitus," in British Journal of Ophthalmology, vol.80, issue 4, pp. 327-331, 1996.

[3] G. Leontidis, B. Al-Diri, A. Hunter, "Diabetic retinopathy: current and future methods for early screening from a retinal hemodynamic and geometric approach," in Expert Review of Ophthalmology, vol.9, issue 5, pp. 431-442, 2014.

[4] T. T. Nguyen, T. Y. Wong, "Retinal vascular changes and diabetic retinopathy," in Current diabetes reports, vol.9, issue 4, pp. 277-283, 2009.

[5] A. Avakian, R. E. Kalina, E. H. Sage, A. H. Rambhia, K. E. Elliott, E. L. Chuang and P. Parsons-Wingerter, "Fractal analysis of regionbased vascular chang in normal and non-proliferative diabetic retina," in Current eye research, vol.24, issue 4, pp. 274-280, 2002.

[6] G. Leontidis, B. Al-Diri, A. Hunter, "Study of the retinal vascular changes in the transition from diabetic to diabetic retinopathy eye," in Engineering in Medicine and Biology Society (EMBC), 36th Annual International Conference of the IEEE. IEEE, 2014.

[7] S. Wang, Y. Yin, G. Cao, B. Wei, Y. Zheung and G. Yang, " Hierarchical retinal blood vessel segmentation based on feature and ensemble learning," in Neurocomputing, vol.149B, pp. 708-717, 2015.

[8] S. Roychowdhury, D. Koozekanani and K. Parhi, "Iterative vessel segmentation of fundus images," in Biomedical Engineering, IEEE Transactions on, vol.PP, no.99, pp.1, 2015.

[9] Y. Zhao, L. Rada, K. Chen, S. Harding and Y. Zheng, “ Automated vessel segmentation using infinite perimeter active contour model with hybrid region information with application to retina images," in Medical Imaging, IEEE Transactions on, vol.PP, no.99, pp.1, 2015.

[10] J. Lowell, A. Hunter, D. Steel, A. Basu, R. Ryder and R. L. Kennedy, "Measurement of retinal vessel widths from fundus images based on 2-D modeling," in Medical Imaging, IEEE Transactions on, vol.23, issue 10, pp.1196-1204, 2004.

[11] P. Bankhead, C. N. Scholfield, J. G. McGeown and T. M. Curtis, "Fast retinal vessel detection and measurement using wavelets and edge detection location refinement," in PLOS ONE, vol.7, issue 3, e32435, 2012.

[12] B. Al-Diri, A. Hunter and D. Steel, "An active contour model for segmenting and measuring retinal vessels," in Medical Imaging, IEEE Transaction on, vol.28, issue 9, pp.1488-1497, 2009.

[13] F. Family, B. R. Masters and D. E. Platt," Fractal pattern formation in human retinal vessels," in Physica D: Nonlinear Phenomena, vol 38, issue 1, pp. 98-103, 1989.

[14] J. Li, Q. Du and C. Sun, "An improved box-counting method for image fractal dimension estimation," in Pattern Recognition, vol.42, issue 11, pp. 2460-2469, 2009.
[15] C. R. Tolle, T. R. McJunkin and D. J. Gorsich, "An efficient implementation of the gliding box lacunarity algorithm," in Physica D: Nonlinear Phenomena, vol. 237, issue 3, pp. 306-315, 2008.

[16] Y. Guo, H. L. Glueck and K. E. Muller, "Selecting a sample size for studies with repeated measures," in BMC medical research methodology, vol.13, issue 1, 100, 2013.

[17] H. Leung, J. J. Wang, E. Rochtchina, T. Y. Wong, R. Klein and P. Mitchell, " Relationships between age, blood pressure, and retinal vessel diameters in older population," in Investigative Ophthalmology \& Visual Science, vol. 44, issue 7, pp. 2900-2904, 2003.

[18] E. R. Girden (ed.)," ANOVA: Repeated measures," in Sage, 1992.

[19] L. Breiman, "Random forests," in Machine learning, vol. 45, issue 1, pp. 5-32, 2001.

[20] A. Liaw and M. Wiener, "Classification and Regression by randomForest," in R News, vol.2, issue 3, pp. 18-22, 2002.

[21] M. D. Knudtson, K. E. Lee, L. D. Hubbard, T. Y. Wong, R. Klein and B. E. Klein," Revised formulas for summarizing retinal vessel diameters," in Current eye research, vol. 27, issue 3, pp. 143-149, 2003.

[22] M. Zamir, "Nonsymmetrical bifurcations in arterial branching," in The Journal of general physiology, vol.72, issue 6, pp. 837-845, 1978. 